\title{
Shared features in the pathobiology of babesiosis and malaria
}

\author{
Peter J. Krause ${ }^{1}$, Johanna Daily ${ }^{2}$, Sam R. Telford ${ }^{3}$, Edouard Vannier ${ }^{4}$, Paul Lantos ${ }^{2}$ \\ and Andrew Spielman ${ }^{2 *}$
}

\author{
${ }^{1}$ University of Connecticut School of Medicine, 263 Farmington Avenue, Farmington, CT 06030, USA \\ ${ }^{2}$ Harvard School of Public Health, 677 Huntington Avenue, Boston, MA 02115, USA \\ ${ }^{3}$ Tufts University School of Veterinary Medicine, 200 Westboro Road, North Grafton, MA 01519, USA \\ ${ }^{4}$ Tufts-New England Medical Center, 750 Washington Street, Boston, MA 02111, USA
}

\begin{abstract}
The pathobiology of malaria has been extensively studied in humans but many questions remain, especially regarding fulminant disease associated with Plasmodium falciparum infection. Babesiosis, recognized since biblical times as an important disease of livestock and more recently as an emerging health problem in humans, is caused by related intraerythrocytic protozoa with a similar pathogenesis and clinical course. Recent studies of cytokine activation and erythrocyte cytoadherence in babesiosis and malaria have exploited these similarities to provide new insights into malaria pathobiology. Continued investigation of similarities and differences in the pathogenesis of babesiosis and malaria should lead to additional fundamental insights for both conditions.
\end{abstract}

\section{A tale of two diseases}

Malaria is a significant cause of morbidity and mortality in humans. Although the pathobiology of this group of diseases has been studied extensively, fundamental questions remain, including issues relating to the pathogenesis of disease caused by Plasmodium falciparum, which accounts for some two million deaths annually. Babesiosis is caused by related intraerythrocytic Babesia protozoa, which elicit a similar inflammatory response and a similar, although generally less severe, clinical course in humans. Murrain, one of the ancient plagues of Egypt and most likely the same disease as the notorious Texas cattle fever, continues to burden cattle breeders in many parts of the world. More recently, human babesiosis has emerged as a worldwide health threat [1-3].

The study of babesial pathogenesis has been recognized as a valuable approach for understanding the pathobiology of $P$. falciparum malaria because of its similarity to malaria and because Babesia spp. infect an array of vertebrate hosts that might serve as disease models for both infections (Table 1). Accordingly, we review those features of babesiosis that are pertinent to the pathogenesis of $P$. falciparum infection and argue that such comparative analyses will continue to provide fertile ground for a better understanding of the full clinical spectrum of both diseases. The term 'pathobiology' is here defined as host

\footnotetext{
Corresponding author: Krause, P.J. (Pkrause@ccmckids.org)

Deceased.

Available online 7 November 2007
}

changes that begin immediately after pathogen transmission. We shall focus on two fundamental pathogenic mechanisms thought to be of central importance in the pathobiology of these infections: (i) erythrocyte cytoadherence; and (ii) the host proinflammatory cytokine response to infection.

\section{Asymptomatic infection}

During the first few weeks following infection by Babesia or Plasmodia spp. in humans, no symptoms are apparent. The parasites initially undergo silent development, the burden within the vasculature has not yet become heavy and the host inflammatory response has not yet developed. Chronic infection tends to be asymptomatic when endogenous mechanisms limit the pathogen load and the immunopathologic response [4]. Asymptomatic Plasmodium infection is common in highly endemic areas where reinfection is frequent and people remain persistently infected. Human babesiosis, a zoonotic infection owing mainly to Babesia microti, is rarely so intensely endemic. This parasite ultimately is cleared in immune-intact human hosts, following an asymptomatic period of infection that can persist for months or years [5]. P. falciparum-immune hosts typically are asymptomatic and clear the infection within days to months, whereas infection by Plasmodium vivax and Plasmodium ovale might not clear for as long as one to four years, and that owing to Plasmodium malariae after 3 to 50 years [6,7]. Between $5 \%$ and $50 \%$ of such malaria infections are asymptomatic in previously infected people $[8,9]$. Around $25 \%$ of adults and $50 \%$ of children who are infected with $B$. microti are asymptomatic [10].

The mechanism for persistent asymptomatic infection is uncertain. Initial work with Babesia argentina (also known as Babesia bovis) and Plasmodium knowlesi showed that antibodies directed against erythrocytes infected by these parasites induced agglutination of infected erythrocytes, and that surface antigenicity varied over time [11,12]. These results suggest that Babesia or Plasmodium spp. infection of erythrocytes causes variable alterations in the erythrocyte membrane. Subsequent work showed that multiple membrane antigenic changes could occur beginning with a single parasite clone, indicating that Babesia and Plasmodium spp. might persist by evading the host immune response through parasite-directed alterations in the erythrocyte membrane [13,14]. Variant antigens 
Table 1. Babesia spp. that share pathobiologic features with Plasmodium spp.

\begin{tabular}{|c|c|c|c|c|c|}
\hline \multirow{2}{*}{ Babesia species } & \multirow{2}{*}{ Host species } & \multicolumn{3}{|c|}{ Pathogenic process investigated } & \multirow{2}{*}{ Refs } \\
\hline & & $\begin{array}{l}\text { Erythrocyte } \\
\text { cytoadherence }\end{array}$ & Cytokine production & Other & \\
\hline B. annae & Dog & & & $x$ & [71] \\
\hline B. bigemina & Cattle & $x$ & & & [37] \\
\hline B. bovis & Cattle & $x$ & $\times$ & $\times$ & {$[11,14,16,17,20,30,41,48,49,54,59,60,68]$} \\
\hline B. caballi & Horse & & $x$ & & {$[66]$} \\
\hline B. canis & Dog & $x$ & & $\times$ & {$[72]$} \\
\hline \multirow[t]{2}{*}{ B. divergens } & Human & $x$ & & $\times$ & {$[22,73]$} \\
\hline & Gerbil & & & $\times$ & [69] \\
\hline \multirow[t]{3}{*}{ B. duncani (WA-1) } & Human & & & $\times$ & [40] \\
\hline & Hamster & $\times$ & $\times$ & & {$[44,45]$} \\
\hline & Mouse & & $\times$ & $\times$ & {$[34,35,46]$} \\
\hline \multirow[t]{3}{*}{ B. microti } & Human & & $\times$ & $\times$ & {$[5,10,28,31,39,58,65]$} \\
\hline & Hamster & & & $\times$ & {$[45]$} \\
\hline & Mouse & & $x$ & & [24] \\
\hline B. ovis & Sheep & & & $\times$ & {$[70]$} \\
\hline B. rodhaini & Mouse & $\times$ & & & [37] \\
\hline
\end{tabular}

expressed by $B$. bovis (variant erythrocyte surface antigen1 or VESA1) and $P$. falciparum ( $P$. falciparum erythrocyte membrane protein1 or PfEMP1) were shown to be encoded by the multigene families ves $1 \alpha$ and var, respectively [15$18]$. The increased genetic variation of the $B$. bovis ves $1 \alpha$ gene family compared with the $P$. falciparum var gene family might result in a greater number of $B$. bovis strains capable of evading the host immune system [16]. The antigenically variant proteins also have been shown to be involved in cytoadhesion of $B$. bovis- and $P$. falciparum-infected erythrocytes to vascular endothelium, although the data are less definitive for VESA than for PfEMP1 (Figure 1). Thus, erythrocyte membrane antigenic variation and cytoadherence seem to be linked.

Piroplasm-induced erythrocyte adherence to vascular endothelium also might facilitate persistent infection, perhaps by diminishing access to host immune cells or preventing circulation through the spleen [18]. Babesia and Plasmodium spp. parasites can mature, replicate, exit the initially infected erythrocyte, and subsequently infect other erythrocytes from the initial adherent endothelial site. Host immune recognition of parasite-derived erythrocyte surface proteins and elimination of one parasite population might lead to expansion of another population that has different adhesive properties, potentially resulting in adherence to different tissue [15]. In a recent study of children living in a highly endemic area for malaria, adherent infected erythrocytes were observed in those hospitalized for severe malaria and in others hospitalized for bacterial pneumonia with silent persistent malarial infection [19]. The intensity of adherence was dependent upon parasite genotype and host tissue location. These data suggest that erythrocyte adherence is important in the perpetuation of $B$. bovis and P. falciparum. Because

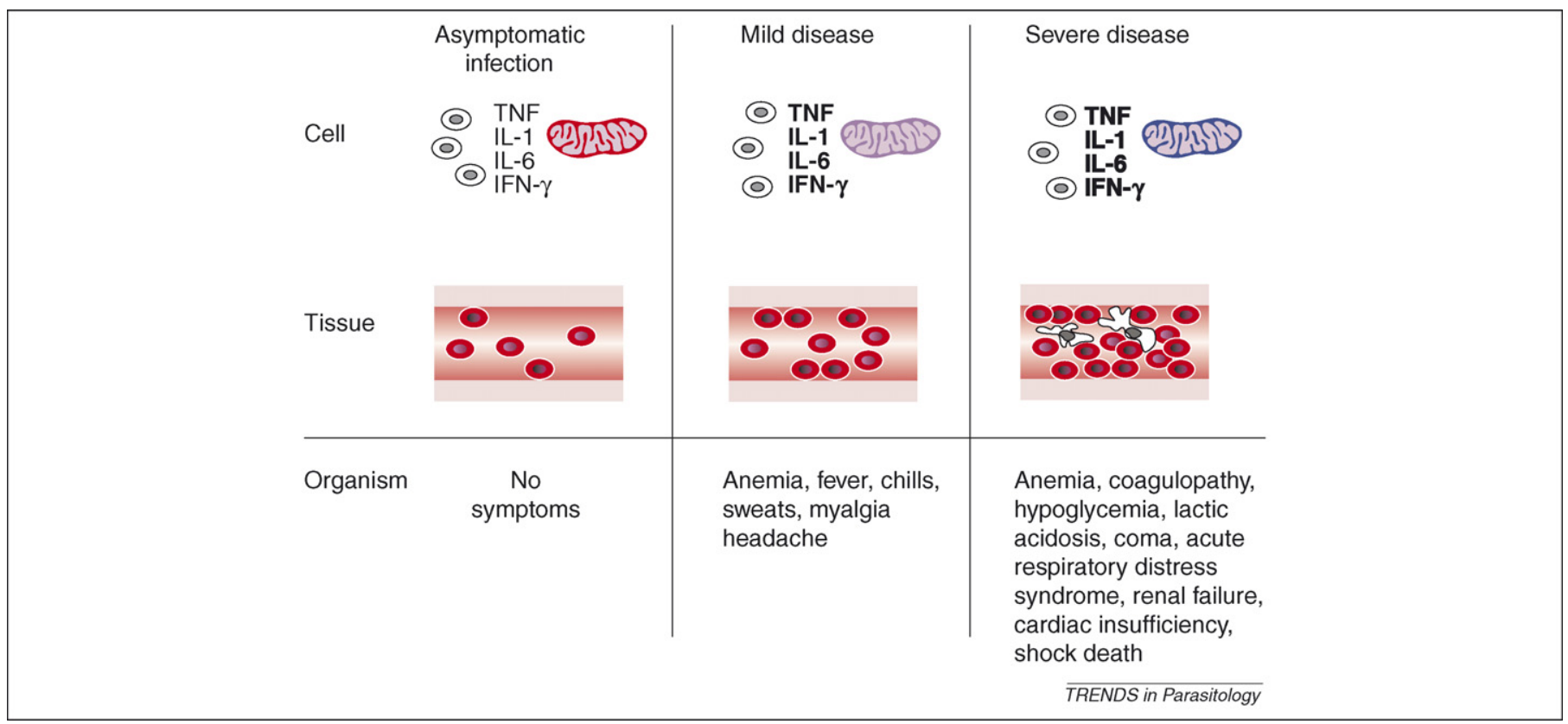

Figure 1. A proposed sequence of pathobiologic events in response to Babesia bovis or Plasmodium falciparum infection is depicted in the cell, tissue and whole organism

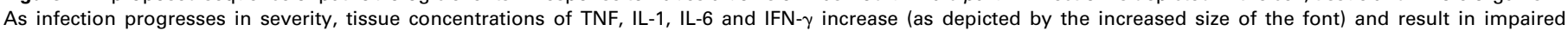
mitochondrial function. Impaired mitochondrial function leads to increased tissue anoxia as depicted by the change in color from red to blue. Increased numbers of infected erythrocytes become adherent to vascular endothelium culminating in erythrocyte sequestration and obstruction of vascular flow by erythrocytes and leukocytes. These events lead to an increase in the number and severity of clinical manifestations. 
many other Babesia and Plasmodium spp. do not adhere, different mechanisms are likely to account for persistent infection. For example, persistent infection could result from failure of the adaptive immune response to clear the pathogen, as noted in a study of $B$. bovis-infected cattle where the untimely release of the anti-inflammatory cytokine IL-10 dampened the host protective effect of interferon gamma (IFN- $\gamma$ ), an activator of nitric oxide production by macrophages [20]. Studies of Babesia and Plasmodium spp. suggest that the net effect of parasiteinduced erythrocyte membrane antigen variability and cytoadherence is long-term asymptomatic infection of the vertebrate host, which increases the probability of transmission to arthropod vectors and to new hosts, thereby assuring pathogen survival.

\section{Moderate disease}

The clinical manifestations of malaria and babesiosis are similar. Indeed, babesiosis is sometimes initially misdiagnosed as malaria until a definitive identification of the pathogen is made on a thin blood smear or by polymerase chain reaction (PCR) $[21,22]$. Most cases of babesiosis and malaria consist of a mild to moderate illness characterized by fever, sweats, chills, headache, myalgia, back and abdominal pain, nausea, vomiting, diarrhea and pallor [6,23]. Although the pathologic events that occur following Babesia and Plasmodium infection are complex and incompletely understood, some evidence indicates that the most important mechanism that accounts for these symptoms is excessive production of proinflammatory cytokines.

Initial work that demonstrated killing of intraerythrocytic B. microti and B. rodhaini in mice pretreated with BCG (bacilli Calmette-Guérin) or Corynebacterium parvum, suggested that a soluble mediator released from macrophages was responsible for pathogen death [24]. Tumor necrosis factor (TNF) was soon identified as a probable candidate and also recognized as causing pathology if produced in excess in mice infected by Plasmodium vinckei [25]. Administration of recombinant TNF to human volunteers induces most of the symptoms of babesiosis and malaria, including fever, sweats, chills, headache, myalgia, abdominal pain, nausea, vomiting and diarrhea [26,27]. Markedly elevated serum concentrations of TNF, IFN- $\gamma$, IL-2, IL-6, E-selectin (expressed in the endothelium), vascular cell adhesion molecule-1 (VCAM-1) and intercellular cell adhesion molecule-1 (ICAM-1) occur during the acute phase of human $B$. microti infection and return to baseline a month after resolution of infection [28]. Evidence has accumulated that whereas moderate production of TNF might help to eradicate Babesia and Plasmodium spp. parasitemia, excessive production of TNF and related proinflammatory cytokines is primarily responsible for symptoms associated with these infections [24,26,29-35].

Arguments have emerged that erythrocytic-associated changes following babesial or malarial infection, including erythrocyte lysis, adherence to endothelial cells and restriction of blood flow in the microvasculature, seem less likely to account for the typical symptoms associated with these diseases $[26,29]$. The synchronous release of Plasmodium spp. from lysed erythrocytes is associated with the classic malarial presentation of paroxysms of fever alternating with periods of fatigue but otherwise relative wellness, a pattern that is seldom noted with babesiosis where parasite release and erythrocyte lysis are non-synchronous. Typical babesial and malarial symptoms, however, do not occur in certain other illnesses associated with erythrocyte lysis [36]. Thus, events associated with erythrocyte lysis (such as release of parasites into the bloodstream), rather than lysis of the erythrocyte itself, seem to be responsible for these symptoms. Although erythrocyte adherence to endothelial cells might occur in mild to moderate malaria, as evidenced by the absence of mature forms of the parasite in the circulation, sequestration of large numbers of erythrocytes with subsequent restriction of blood flow to microvessels has only been linked with severe malarial disease and is absent in people experiencing asymptomatic persistent malaria [26]. Similarly, erythrocyte adherence occurs in cattle that are chronically infected by Babesia bigemina but remain asymptomatic [37]. Consequently, erythrocyte adherence alone probably does not account for symptoms associated with malaria and babesiosis. Studies of babesiosis and malaria have indicated that moderate illness cannot be explained solely by erythrocyte-associated pathologic changes and have suggested an important role for cytokines in their pathogenesis.

\section{Severe disease}

Severe $P$. falciparum disease is defined as a syndrome that includes a complex array of metabolic abnormalities and organ dysfunction [38]. P. falciparum generally causes more severe disease than the other species of Plasmodium (P. vivax, $P$. malariae, $P$. ovale) or than the Babesia species that infect humans (B. divergens, $B$. duncani [Washington1 or WA1], B. microti, EU1 [European Union1], MO1 [Missouri1], and TW1 [Taiwan1]). A pattern of disease similar to that of malaria might occur, however, in people experiencing severe babesiosis [22,39,40]. Although individual manifestations are described as discrete entities, patients experiencing fulminant $P$. falciparum malaria or babesiosis might suffer simultaneous complications of several organ systems with similar pathologic findings [6,41]. Furthermore, whereas any non-immune person is at risk of fulminant $P$. falciparum disease, severe babesial illness almost always occurs in people who are immunosuppressed with heavy parasitemia, including those with advanced age, asplenia, malignancy or HIV coinfection [42].

The pathogenesis of severe $P$. falciparum infection is complex and incompletely understood, but major pathologic events include: (i) overproduction of inflammatory cytokines triggered by the parasite; (ii) microvascular obstruction and tissue hypoxia caused by adherence of erythrocytes to vascular endothelium; (iii) anemia and tissue anoxia caused by lysis of erythrocytes; (iv) hypoglycemia and lactic acidemia caused by anaerobic metabolism of glucose; (v) tissue hemorrhage owing to alteration in hemostasis; and (vi) cerebral, pulmonary, renal and hepatic impairment caused by the cumulative effects of these pathologic processes [6,26].

The same pathogenic mechanisms associated with mild to moderate disease are also thought to contribute to 
severe manifestations of babesiosis and malaria (Figure 1). There seems to be a continuum of intensity of cytokine activation and erythrocyte cytoadherence-sequestration, which depends on parasite species and strain, and host immunity at both the general and local tissue level. In particular, an array of pathologic events has been associated with excessive proinflammatory cytokine release. Both TNF and IL-1 increase the expression of adhesion molecules at the surface of vascular endothelium, including ICAM, VCAM, and P-selectin (expressed on vascular endothelium and platelets), and induce the generation of nitric oxide [38]. Nitric oxide has both homeostatic and pathogenic effects. It can inhibit mitochondrial respiration and the $\mathrm{Na}^{+} / \mathrm{K}^{+}$pump, which can lead to decreased erythrocyte deformity and to parasite killing $[26,43]$. In both Babesia and Plasmodium spp. infection, proinflammatory cytokine release can induce nitric oxide production $[26,30,43]$. In sum, intense immunologic activation creates an inflammatory milieu that promotes erythrocyte sequestration, hypoglycemia, metabolic acidosis, hyponatremia, coagulopathy and thrombocytopenia $[26,29]$. The end result is similar pathology in the brain, lung, kidney and other organs [6,19,22,44-46].

\section{Central nervous system complications}

Cerebral malaria is one of the most lethal manifestations of $P$. falciparum infection and occurs in $\sim 10 \%$ of hospitalized cases with a $20 \%$ mortality rate [47]. Although a similar syndrome in human babesiosis is poorly described, cerebral pathology as a result of $B$. bovis infection is a well-recognized complication in cattle and has been used as an animal model of cerebral malaria $[16,48,49]$.

Erythrocyte cytoadherence and sequestration generally are found in the cerebral vasculature of patients dying of cerebral malaria [19,50-53]. Several studies of $B$. bovis cerebral disease have demonstrated extensive erythrocyte cytoadherence and sequestration in the brains of infected animals $[48,49,51,52]$. Stellate protrusions have been described from $B$. bovis-infected erythrocytes that are similar to the knobs seen in $P$. falciparum and that seem to mediate erythrocyte adherence to vascular endothelium and other erythrocytes [49,52,54]. Erythrocyte cytoadherence and sequestration also are described in the lung, kidney and liver, although to a lesser extent than in the brain. Similar findings also have been described in the lungs of hamsters and mice infected by B. duncani [44-46].

It has been argued that the fundamental cause of cerebral malaria and other complications of $P$. falciparum infection is excessive production of proinflammatory cytokines by the host in response to infection $[25,26,29]$. Other pathologic processes are thought to ensue, including erythrocyte adherence and sequestration $[26,29]$. TNF has been shown to upregulate ICAM-1 and VCAM-1 endothelial cell surface molecules, which might serve as receptors for leukocytes and $P$. falciparum-infected erythrocytes. Elevated TNF blood concentrations have been associated with lethal outcomes in children who have cerebral malaria [32]. TNF and upregulated endothelial surface molecules have been demonstrated in post-mortem brain tissue in 14 Ghanian children who died of malaria, and a child who died from salmonella septicemia and malaria co-infection
[55]. The upregulation of these adhesion molecules colocalized with erythrocyte sequestration; however, there was no difference in upregulation in those who died of cerebral malaria or severe malarial anemia. TNF and IL-1 were detected in and around vascular endothelium in all cases [55]. Elevated levels of TNF alone, however, are unlikely to fully explain symptoms that characterize $P$. falciparum cerebral malaria [56].

Proinflammatory cytokine activation seems to promote adhesion and sequestration of infected erythrocytes, but might cause cerebral pathology in $P$. falciparum malaria through additional mechanisms. For example, mitochondrial dysfunction could mimic the pathologic effects of poor oxygen supply that can occur with erythrocyte sequestration and vascular obstruction $[26,29,57]$. Patients experiencing cerebral malaria often have a relatively rapid recovery time without any evidence of permanent sequelae, an observation that does not fit with the erythrocyte sequestration-stroke model of cerebral pathogenesis [26]. Swelling and vascular congestion of the brain and other organs were demonstrated in a fatal human case of $B$. divergens, and although most cerebral vessels were distended with cells there was no sequestration [22]. Recently, the microvasculature of brain sections from a comatose patient who died of $B$. microti infection was shown to lack adherent erythrocytes [58]. These data suggest that cerebral manifestations of human babesiosis can be independent of erythrocyte sequestration and, by inference, at least some cases of cerebral malaria as well $[29,58]$. Interestingly, in vitro and in vivo studies in cattle demonstrated that erythrocyte adherence to vascular endothelium by different $B$. bovis strains is not associated with strain virulence, suggesting that the ability of peripheral erythrocytes to adhere to endothelial cells is not of crucial importance in severe $B$. bovis infections $[51,59,60]$. By contrast, more recent work that examined erythrocyte adherence under conditions of physiologic blood flow has indicated that more virulent strains of $B$. bovis are associated with increased adherence [54,61].

\section{Lung complications}

Pulmonary complications are often observed in adults experiencing severe malaria. Hypoxemia is associated with an array of pathologic processes, which include the host inflammatory response, erythrocyte sequestration in the microvasculature, severe anemia, intravascular depletion, heart failure, non-cardiac pulmonary edema, or secondary bacterial sepsis and pneumonia [62]. Intravascular sequestration of parasites and leukocytes has been reported on autopsy specimens of lung [63,64]. Pulmonary disease is the most common complication in people experiencing severe babesial infection with up to $20 \%$ of patients suffering from non-cardiac pulmonary edema $[39,65]$. The role of proinflammatory cytokines in the pathogenesis of pulmonary complications of $B$. duncani infection has been studied in a mouse model $[34,35,46]$. These mice suffer a fulminant illness that includes increased leukocyte margination in lung vasculature, pulmonary edema and death, but mice infected by $B$. microti experience few symptoms and easily recover $[34,35,46]$. TNF- $\alpha$ and IFN- $\gamma$ are upregulated in the lungs of $B$. duncani-infected mice, but not in those of $B$. 
microti-infected mice [34]. Mice with a genetic disruption in the TNF pathway are less likely to die of fulminating $B$. duncani infection than mice with an intact TNF response [35]. In another animal model, horses infected by $B$. caballi and dying of pulmonary edema have increased levels of IL-10, IFN- $\gamma$ and iNOS (inducible nitric oxide synthase) in lung tissue [66]. These studies point to the central role of cytokines, and especially $\mathrm{TNF}$, in babesia and malaria pulmonary pathogenesis.

\section{Kidney complications}

Renal impairment is a common complication of P. falciparum and babesial infections. Renal failure has been reported in $\sim 5 \%$ of severe $B$. microti human infection and even more frequently in patients experiencing severe B. duncani infection $[39,40]$. On autopsy of patients dying of malaria, several distinct pathologic patterns are noted in the kidneys, with erythrocyte sequestration and exposure to products of lysed red blood cells contributing to the pathology [67]. Studies in cattle infected with $B$. bovis and hamsters infected with $B$. duncani have demonstrated erythrocyte sequestration and evidence of vascular stasis $[44,46,68]$. Renal impairment has been studied in other animals including $B$. divergens in gerbils, Babesia ovis in sheep, and Babesia annae and Babesia canis in dogs [6972]. The role of cytokine activation on renal pathology in babesiosis and malaria has not been investigated.

\section{Perspective}

The first studies of the pathogenesis of malaria were initiated more than a century ago but our understanding of the complex pathobiology of this disease remains incomplete. Because Babesia spp. share unique similarities with Plasmodium spp. they offer exceptional opportunities for providing a better understanding of the pathogenesis of malaria. Important advances regarding malarial pathobiology have been made with studies of Babesia spp. alone, and with comparative studies between Babesia and Plasmodium, especially $B$. bovis and $P$. falciparum. Although the prospect for large-scale human $B$. duncani studies might be tempered by the apparently limited number of human cases, important information can be derived from comparative observations of only a few such cases and those in animals. By contrast, hundreds of people are infected by $B$. microti every year in the USA and as many as $10 \%$ of these cases are admitted to hospital with severe disease. As the number of cases of human babesiosis continues to increase, further opportunities arise for investigation of the pathogenesis of these cases and comparison with animal models of disease. Continued investigation of similarities and differences in the pathogenesis of babesiosis and malaria should lead to additional fundamental insights for both conditions.

\section{Acknowledgements}

We dedicate this review to the memory of Dr. Andrew Spielman who died shortly before its completion. Beloved teacher, mentor and friend; he is sorely missed. This study was supported by Grant M01 RR06192 (P.J.K.) from the National Center for Research Resources (NCRR), a component of the National Institutes of Health (NIH). Its contents are solely the responsibility of the authors and do not necessarily represent the official view of NCRR or NIH.

\section{References}

1 Lederberg, J. et al., eds (1992) Emerging Infections: Microbial Threats to Health in the United States, National Academy Press

2 Gorenflot, A. et al. (1998) Human babesiosis. Ann. Trop. Med. Parasitol. 92, 489-501

3 Homer, M.J. et al. (2000) Babesiosis. Clin. Microbiol. Rev. 13, 451-469

4 Boutlis, C.S. et al. (2006) Malaria tolerance - for whom the cell tolls? Trends Parasitol. 22, 371-377

5 Krause, P.J. et al. (1998) Persistent parasitemia following acute babesiosis. N. Engl. J. Med. 339, 160-165

6 Taylor, T.E. and Strickland, G.T. (2000) Malaria. In Hunter's Tropical Medicine and Emerging Infectious Diseases (8th edn) (Strickland, G.T., ed.), pp. 614-643, W.B. Saunders

7 Nsobya, S.L. et al. (2004) Molecular evaluation of the natural history of asymptomatic parasitemia in Ugandan children. J. Infect. Dis. 189, $2220-2226$

8 Alves, F.P. et al. (2002) High prevalence of asymptomatic Plasmodium vivax and Plasmodium falciparum infections in native Amazonian populations. Am. J. Trop. Med. Hyg. 66, 639-640

9 Coura, J.R. et al. (2006) A new challenge for malaria control in Brazil: asymptomatic Plasmodium infection- a review. Mem. Inst. Oswaldo Cruz 101, 229-237

10 Krause, P.J. et al. (2003) Increasing health burden of human babesiosis in endemic sites. Am. J. Trop. Med. Hyg. 68, 431-436

11 Curnow, J.A. (1968) In vitro agglutination of bovine erythrocytes infected with Babesia argentina. Nature 217, 267-268

12 Brown, K.N. and Brown, I.N. (1965) Immunity to malaria: antigenic variation in chronic infections of Plasmodium knowlesi. Nature 208, 1286-1288

13 Barnwell, J.W. et al. (1983) Splenic requirement for antigenic variation and expression of the variant antigen on the erythrocyte membrane in cloned Plasmodium knowlesi malaria. Infect. Immun. 40, 985-994

14 Curnow, J.A. (1973) Studies on antigenic changes and strain differences in Babesia argentina infections. Aust. Vet. J. 49, 279-283

15 Allred, D.R. (2003) Babesiosis: persistence in the face of adversity. Trends Parasitol. 19, 51-55

16 Allred, D.R. and Al-Khedery, B. (2004) Antigenic variation and cytoadhesion in Babesia bovis and Plasmodium falciparum: different logics achieve the same goal. Mol. Biochem. Parasitol. 134, 27-35

17 Al-Khedery, B. and Allred, D.R. (2006) Antigenic variation in Babesia bovis occurs through segmental gene conversion of the ves multigene family, within a bidirectional locus of active transcription. Mol. Microbiol. 59, 402-414

18 Kyes, S. et al. (2001) Antigenic variation at the infected red cell surface in malaria. Annu. Rev. Microbiol. 55, 673-707

19 Montgomery, J. et al. (2006) Genetic analysis of circulating and sequestered populations of Plasmodium falciparum in fatal pediatric malaria. J. Infect. Dis. 194, 115-122

20 Goff, W.L. et al. (2001) The age-related immunity in cattle to Babesia bovis infection involves the rapid induction of interleukin-12, interferon-gamma and inducible nitric oxide synthatase mRNA expression in the spleen. Parasite Immunol. 23, 463-471

21 Wittner, M. et al. (1982) Successful chemotherapy of transfusion babesiosis. Ann. Intern. Med. 96, 601-604

22 Fitzpatrick, J.E.P. et al. (1969) Further details of third recorded case of Redwater (Babesiosis) in man. BMJ 4, 770-772

23 Krause, P.J. et al. (1996) Concurrent Lyme disease and babesiosis. Evidence for increased severity and duration of illness. J. Am. Med. Assoc. 275, 1657-1660

24 Clark, I.A. et al. (1976) Protection of mice against Babesia and Plasmodium with BCG. Nature 259, 309-311

25 Clark, I.A. et al. (1981) Possible importance of macrophage-derived mediators in acute malaria. Infect. Immun. 32, 1058-1066

26 Clark, I.A. et al. (2004) Pathogenesis of malaria and clinically similar conditions. Clin. Microbiol. Rev. 17, 509-539

27 Creagan, E.T. et al. (1988) A phase I clinical trial of recombinant human tumor necrosis factor. Cancer 62, 2467-2471

28 Shaio, M.F. and Lin, P.R. (1998) A case study of cytokine profiles in acute human babesiosis. Am. J. Trop. Med. Hyg. 58, 335-337

29 Clark, I.A. et al. (2006) Human malarial disease: a consequence of inflammatory cytokine release. Malar. J. 5, 85

30 Shoda, L.K. et al. (2000) Babesia bovis-stimulated macrophages express interleukin-1beta, interleukin-12, tumor necrosis factor 
alpha, and nitric oxide and inhibit parasite replication in vitro. Infect. Immun. 68, 5139-5145

31 Taiwo, B. et al. (2007) Can tumor necrosis factor $\alpha$ blockade predispose to severe babesiosis? Arthritis Rheum. 57, 179-181

32 Kwiatkowski, D. et al. (1990) TNF concentration in fatal cerebral, nonfatal cerebral, and uncomplicated Plasmodium falciparum malaria. Lancet 336, 1201-1204

33 Karunaweera, N.D. et al. (1992) Tumour necrosis factor-dependent parasite-killing effects during paroxysms in non-immune Plasmodium vivax malaria patients. Clin. Exp. Immunol. 88, 499-505

34 Hemmer, R.M. et al. (2000) Up-regulation of tumor necrosis factoralpha and interferon-gamma expression in the spleen and lungs of mice infected with the human Babesia isolate WA1. Parasitol. Res. 86, 121128

35 Hemmer, R.M. et al. (2000) Role of T cells and cytokines in fatal and resolving experimental babesiosis: protection in TNFRp55-/- mice infected with the human Babesia WA1 parasite. J. Parasitol. 86, 736742

36 Pruss, A. et al. (2003) Immune hemolysis-serological and clinical aspects. Clin. Exp. Med. 3, 55-64

37 Parrodi, F. et al. (1990) In vitro adherence of erythrocytes infected with Babesia bigemina and Babesia rodhaini to thrombospondin. Int. J. Parasitol. 20, 899-903

38 Severe falciparum malaria. World Health Organization, Communicable Diseases Cluster (2000). Trans. R. Soc. Trop. Med. Hyg. 94, S1-S90

39 Hatcher, J.C. et al. (2001) Severe babesiosis in Long Island: review of 34 cases and their complications. Clin. Infect. Dis. 32, 1117-1125

40 Persing, D.H. et al. (1995) Infection with a Babesia-like organism in northern California. N. Engl. J. Med. 332, 298-303

41 Brown, W.C. et al. (2006) Immune control of Babesia bovis infection. Vet. Parasitol. 138, 75-87

42 Krause, P.J. et al. (2007) Persistent and relapsing Babesiosis in immunocompromised patients. Clin. Infect. Dis. (in press)

43 Clark, I.A. and Cowden, W.B. (2003) The pathophysiology of falciparum malaria. Pharmacol. Ther. 99, 221-260

44 Dao, A.H. and Eberhard, M.L. (1996) Pathology of acute fatal babesiosis in hamsters experimentally infected with the WA-1 strain of Babesia. Lab. Invest. 74, 853-859

45 Wozniak, E.J. et al. (1996) Comparative pathogenesis of human WA-1 and Babesia microti isolates in a Syrian hamster model. Lab. Anim. Sci. 46, 507-515

46 Hemmer, R.M. et al. (1999) Endothelial cell changes are associated with pulmonary edema and respiratory distress in mice infected with the WA1 human Babesia parasite. J. Parasitol. 85, 479-489

47 Idro, R. et al. (2005) Pathogenesis, clinical features, and neurological outcome of cerebral malaria. Lancet Neurol. 4, 827-840

48 Everitt, J.I. et al. (1986) Experimental Babesia bovis infection in Holstein calves. Vet. Pathol. 23, 556-562

49 O'Connor, R.M. et al. (1999) Cytoadherence of Babesia bovis-infected erythrocytes to bovine brain capillary endothelial cells provides an in vitro model for sequestration. Infect. Immun. 67, 3921-3928

50 Clark, I.A. et al. (2003) Tissue distribution of migration inhibitory factor and inducible nitric oxide synthetase in falciparum malaria and sepsis in African children. Malar. J. 2, 6

51 Wright, I.G. et al. (1988) Immunopathophysiology of Babesia bovis and Plasmodium falciparum infections. Parasitol. Today 4, 214-218

52 Schetters, T.P. and Eling, W.M. (1999) Can Babesia infections be used as a model for cerebral malaria? Parasitol. Today 15, 492-497
53 Taylor, T.E. et al. (2004) Differentiating the pathologies of cerebral malaria by postmortem parasite counts. Nat. Med. 10, 143-145

54 Hutchings, C.L. et al. (2007) New insights into the altered adhesive and mechanical properties of red blood cells parasitized by Babesia bovis. Mol. Microbiol. 65, 1092-1105

55 Armah, H. et al. (2005) Cytokines and adhesion molecules expression in the brain in human cerebral malaria. Int. J. Environ. Res. Public Health 2, 123-131

56 Karunaweera, N.D. et al. (1992) Dynamics of fever and serum levels of tumor necrosis factor are closely associated during clinical paroxysms in Plasmodium vivax malaria. Proc. Natl. Acad. Sci. U. S. A. 89, 32003203

57 Thurnham, D.I. et al. (1971) The inhibition of mitochondrial respiration and oxidative phosphorylation by serum from malariainfected animals. II. The inhibitory activity of serum ultrafiltrates from Plasmodium knowlesi-infected monkeys. Ann. Trop. Med. Parasitol. 65, 287-295

58 Clark, I.A. et al. (2006) Absence of erythrocyte sequestration in a case of babesiosis in a splenectomized human patient. Malar. J. 5, 69

59 Molloy, J.B. et al. (2003) Babesia bovis: adhesion of parasitized red blood cells to bovine umbilical vein endothelial cells in vitro does not select for virulence. Exp. Parasitol. 103, 182-184

60 Canto, G.J. et al. (2006) Evaluation of cattle inoculated with Babesia bovis clones adhesive in vitro to bovine brain endothelial cells. Ann. N. Y. Acad. Sci. 1081, 397-404

61 Cooke, B.M. et al. (2005) Cellular adhesive phenomena in apicomplexan parasites of red blood cells. Vet. Parasitol. 132, 273-295

62 Taylor, W.R. et al. (2006) Pulmonary manifestations of malaria: recognition and management. Treat. Respir. Med. 5, 419-428

63 Dobano, C. et al. (2007) Expression of merozoite surface protein markers by Plasmodium falciparum-infected erythrocytes in peripheral blood and tissues of children with fatal malaria. Infect. Immun. 75, 643-652

64 Seydel, K.B. et al. (2006) The distribution and intensity of parasite sequestration in comatose Malawian children. J. Infect. Dis. 194, 208 215

65 White, D.J. et al. (1998) Human babesiosis in New York State: review of 139 hospitalized cases and analysis of prognostic factors. Arch. Intern. Med. 158, 2149-2154

66 Hanafusa, Y. et al. (1998) Pathogenesis of Babesia caballi infection in experimental horses. J. Vet. Med. Sci. 60, 1127-1132

67 Stone, W.J. et al. (1972) Acute renal insufficiency due to falciparum malaria. Review of 42 cases. Arch. Intern. Med. 129, 620-628

68 Patarroyo, J.H. et al. (1982) Description of lesions in cattle in a natural outbreak of Babesia bovis infection in Brazil. Vet. Parasitol. 11, 301308

69 Liddell, K.G. et al. (1981) Babesia divergens infections in the Mongolian gerbil: characteristics of a human strain. Parasitology $82,205-224$

70 Habela, M.A. et al. (1991) Histopathological changes in sheep experimentally infected with Babesia ovis. Vet. Parasitol. 38, 1-12

71 Camacho, A.T. et al. (2005) Serum protein response and renal failure in canine Babesia annae infection. Vet. Res. 36, 713-722

72 Reyers, F. et al. (1998) Canine babesiosis in South Africa: more than one disease. Does this serve as a model for falciparum malaria? Ann. Trop. Med. Parasitol. 92, 503-511

73 Zintl, A. et al. (2003) Babesia divergens, a bovine blood parasite of veterinary and zoonotic importance. Clin. Microbiol. Rev. 16, 622636 